\title{
343 - Best Practice Guidance on Human Interaction with Technology in Dementia - Recommendations from the INDUCT Network
}

Rose-Marie Dröes ${ }^{1}$, Yvette Vermeer ${ }^{2}$, Sébastien Libert ${ }^{2}$, Sophie Gaber ${ }^{3}$, Sarah Wallcook ${ }^{3}$, Harleen Rai ${ }^{4}$, Aline Cavalcanti Barroso ${ }^{4}$, Joeke van Santen ${ }^{1}$, Floriana Mangiaracina ${ }^{1}$, Kim Beentjes $^{1}$, Sara Bartels ${ }^{5}$, Hannah Christie ${ }^{5}$, Rose Miranda ${ }^{6}$, Annelien van Dael ${ }^{6}$, Kate Shiells ${ }^{7}$, Ángel C. Pinto Bruno ${ }^{8}$, Angie Alejandra Diaz ${ }^{9}$, Lieve Van den Block ${ }^{6}$, Lara Pivodic ${ }^{6}$, Louise Nygard ${ }^{3}$, Manuel Franco Martin ${ }^{9}$, Paul Higgs ${ }^{2}$, Iva Holmerova ${ }^{7}$, Camilla Malinowsky ${ }^{3}$, Franka Meiland $^{1}$, Henriëtte van der Roest ${ }^{9}$, Justine Schneider ${ }^{4}$, Annemieke van Straten ${ }^{8}$, Frans Verhey ${ }^{5}$, Marjolein de Vugt ${ }^{5}$, Martin Orrell ${ }^{4}$

${ }^{1}$ VU University Medical Centre, Amsterdam, Netherlands, ${ }^{2}$ University College London, London, United Kingdom, ${ }^{3}$ Karolinska Institute, Stockholm, Sweden ${ }^{4}$ University of Nottingham, Nottingham, United Kingdom, ${ }^{5}$ Alzheimer Center Limburg/Maastricht University, Maastricht, the Netherlands, ${ }^{6}$ Vrije Universiteit Brussel, Brussel, Belgium, ${ }^{7}$ Charles University, Prague, Czech Republic, ${ }^{8}$ Vrije Universiteit, Amsterdam The Netherlands, ${ }^{9}$ Universidad de Salamanca, Salamanca, Spain

The Interdisciplinary Network for Dementia Using Current Technology, INDUCT, is a Marie Sklodowska Curie funded International Training Network that aims to develop a multi-disciplinary, inter-sectorial educational research framework for Europe to improve technology and care for people with dementia, and to provide the evidence to show how technology can improve the lives of people with dementia. Within INDUCT (2016-2020) 15 Early Stage Researchers worked on projects in the areas of Technology to support every day life; technology to promote meaningful activities; and health care technology.

Three transversal objectives were adopted by INDUCT: 1) To determine the practical, cognitive and social factors needed to make technology more useable for people with dementia; 2) To evaluate the effectiveness of specific contemporary technology; and 3) To trace facilitators and barriers for implementation of technology in dementia care.

The main recommendations resulting from the research projects are integrated in a web-based digital Best Practice Guidance on Human Interaction with Technology in Dementia which will be presented at the congress. The recommendations are meant to be helpful for different target groups, i.e. people with dementia, their formal and informal carers, policy makers, designers and researchers, who can easily select the for them relevant recommendations in the Best Practice Guidance by means of a selection tool. The main aim of the Best Practice Guidance is to improve the development, usage and implementation of technology for people with dementia in the three mentioned technology areas.

This Best Practice Guidance is the result of the intensive collaborative partnership of INDUCT with academic and non-academic partners as well as the involvement of representatives of the different target groups throughout the INDUCT project.

Acknowledgements: The research presented was carried out within the Marie Sklodowska Curie International Training Network (ITN) action, H2020-MSCA-ITN-2015, grant agreement number 676265. 